\title{
Nonlinear Vibration Model for Initially Stressed Beam-Foundation System
}

\author{
M.H. Taha* \\ Department of Engineering Mathematics and Physics, Faculty of Engineering, Cairo University, Giza, Egypt
}

\begin{abstract}
An analytical solution for nonlinear vibration of an initially stressed beam with elastic end restraints resting on a nonlinear elastic foundation is obtained. As a first step in solving nonlinear vibration equation, the linear vibration mode functions for a beam with elastic end restraints resting on a linear elastic foundation are obtained. Then, the nonlinear vibration equation is solved by employing the linear mode functions to obtain frequency equation and nonlinear response using Jacobi elliptic integral. The nonlinearity due to lateral vibrations, the nonlinearity of foundations and lateral displacement due to lateral elastic restraints at beam ends not included in previous analytical work are considered in the present work. The effects of spring stiffness at the beam ends, foundation stiffness, axial load and vibration amplitude on the frequency parameter are studied. The present solution can be used to measure the accuracy of approximate methods.
\end{abstract}

Keywords: Nonlinear beam vibration, elliptic integrals, nonlinear foundations, mode functions and natural frequencies.

\section{INTRODUCTION}

Many practical engineering applications as railroad tracks, highway pavement, buried pipelines and foundation beams are modeled as beams resting on elastic foundations. To investigate the dynamics of the vibrational behavior of these applications, solutions need to be obtained. Few analytical solutions, limited to idealized cases for vibrations of such models are found in the literature. This is due to the intractable mathematical nature of the problem. Numerical methods such as finite element method [1-2], transfer matrix method [3], differential quadrature element method (DQM) [4-6], perturbation techniques [7-8] are used to obtain the vibration behavior of different types of linear or nonlinear beams resting on linear or nonlinear foundations.

Semi-analytical methods, such as series solutions, are suggested to obtain frequencies and mode functions of nonuniform beams resting on elastic foundation $[9,10]$. Taha M.H. [11] studied the transient response of beams resting on viscoelastic foundation under stochastic dynamic loads. Taha M.H. and Abohadima S. [12] analyzed the vibrational behavior of a nonuniform flexural beam using Bessel's functions. Abohadima, S. and Taha M.H. [13] extended their works to the vibrational behavior of a nonuniform beam resting on a nonuniform foundation.

However, in most studies, the boundary conditions assumed to simulate the actual conditions at beam supports are idealized to obtain simple solutions. In fact, there is no absolutely clamped or pinned support, actually all supports always allow some movements. Also, few researchers studied the effects of axial load on the vibration behavior of such beams and nonlinearity due to stretching resulting from lateral vibrations.

*Address correspondence to this author at the Department of Engineering Mathematics and Physics, Faculty of Engineering, Cairo University, Giza, Egypt; Tel: +20-2-37348595; Fax: +20-2-37348594;

E-mail: mtaha@alfaconsult.org
In the present work, the effects of above mentioned parameters are taken into account. The nonlinear vibration of an initially stressed beam with elastic end restraints resting on a nonlinear elastic foundation is solved using elliptic integrals. The obtained solutions are verified against those obtained from numerical methods and found in close agreement. Parametric study to investigate the influences of foundation stiffness, elastic end restraints stiffnesses, initial axial load and vibration amplitude are conducted and results are depicted in graphs for a wide range of the different practical characteristics.

\section{ANALYSIS}

\subsection{Vibration Equation}

The equation of motion of a uniform beam with elastic end restrains, initially stressed by an axial load $P_{o}$, resting on a nonlinear elastic foundation, shown in Fig. (1) is given as:

$$
\begin{aligned}
& E I \frac{\partial^{4} Y}{\partial X^{4}}+P_{o} \frac{\partial^{2} Y}{\partial X^{2}}+\mu \frac{\partial^{2} Y}{\partial t^{2}}-\frac{E A}{2 L}\left\{\int_{0}^{L}\left(\frac{\partial Y}{\partial X}\right)^{2} d X\right\} \frac{\partial^{2} Y}{\partial X^{2}}+ \\
& k_{1} Y(X, t)+k_{2} Y^{3}(X, t)=0
\end{aligned}
$$

where $E I$ is the flexural stiffness of the beam, $L$ is the beam length, $\mu$ is the beam mass per unit length, $k_{1}$ and $k_{2}$ are the foundation stiffnesses coefficients per unit length, $E$ is the modulus of elasticity of the beam, $A$ is the area of the beam cross section, $Y(X, t)$ is the lateral response of the beam, $X$ is the coordinate along the beam and $t$ is time.

Using the dimensionless parameters $x=X / L$ and $y=Y / L$, eqn. (1) may be rewritten as:

$$
\begin{aligned}
& \frac{E I}{\mu L^{4}} \frac{\partial^{4} y}{\partial x^{4}}+\frac{P_{o}}{\mu L^{2}} \frac{\partial^{2} y}{\partial x^{2}}+\frac{\partial^{2} y}{\partial t^{2}}-\frac{E A}{2 \mu L^{2}}\left\{\int_{0}^{1}\left(\frac{\partial y}{\partial x}\right)^{2} d x\right\} \frac{\partial^{2} y}{\partial x^{2}}+ \\
& \frac{k_{1}}{\mu} y(x, t)+\frac{k_{2} L^{2}}{\mu} y^{3}(x, t)=0
\end{aligned}
$$

The solution of the nonlinear partial differential eqn. (2) is obtained by employing the linear mode functions and 
integrating over the domain of the dimensionless spatial variable $x$ to separate the time variation. However, the solution of eqn. (2) depends on the boundary conditions at beam ends.

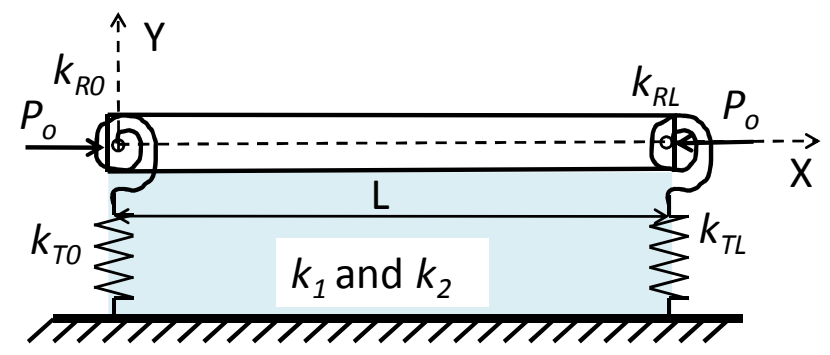

Fig. (1). Initially stressed beam-foundations system.

\subsection{Boundary Conditions}

The boundary conditions due to elastic end restraints at $x=0$ are given as;

$$
\begin{aligned}
& k_{T 0} y(0, t)=-\frac{E I}{L^{3}} \frac{\partial^{3} y(0, t)}{\partial x^{3}}, \\
& k_{R 0} \frac{\partial y(0, t)}{\partial x}=\frac{E I}{L} \frac{\partial^{2} y(0, t)}{\partial x^{2}} .
\end{aligned}
$$

and at $x=1$ are:

$k_{T L} y(1, t)=\frac{E I}{L^{3}} \frac{\partial^{3} y(1, t)}{\partial x^{3}}$,

$k_{R L} \frac{\partial y(1, t)}{\partial x}=-\frac{E I}{L} \frac{\partial^{2} y(1, t)}{\partial x^{2}}$.

where $k_{T 0}$ and $k_{T L}$ are the elastic stiffnesses of lateral restraints at $x=0,1.0$ respectively and $k_{R 0}$ and $k_{R L}$ are the elastic rotation stiffnesses of beam support at $x=0,1.0$ respectively.

\subsection{Solution of Linear Vibration Equation}

A linear version of eqn. (2), neglecting the initial axial load, can be expressed as:

$$
\frac{E I}{\mu L^{4}} \frac{\partial^{4} y}{\partial x^{4}}+\frac{\partial^{2} y}{\partial t^{2}}+\frac{k_{1}}{\mu} y(x, t)=0
$$

Following the separation of variables analogy, the solution of eqn. (4) may be assumed as:

$$
y(x, t)=y_{o} \phi(x) \psi(t)
$$

where $\phi(x)$ is the linear mode function, $\psi(t)$ is a function representing the variation of the response with time and $y_{o}$ is the dimensionless vibration amplitude (obtained from the initial conditions). Substituting eqn. (5) into eqn.(4), eqn.(4) is separated into:

$$
\begin{aligned}
& \left.\frac{d^{4} \phi}{d x^{4}}\right)-\lambda_{f}^{4} \phi(x)=o \\
& \frac{d^{2} \psi}{d t^{2}}+\omega^{2} \psi(t)=0
\end{aligned}
$$

where $\omega$ is the separation constant which represents the natural frequency and $\lambda_{f}$ is the frequency parameter which is given as:

$$
\lambda_{f}^{4}=\frac{\mu L^{4}}{E I}\left(\omega^{2}-\frac{k_{1}}{\mu}\right)
$$

The general solution of eqn. (6) is given as:

$$
\phi(x)=C_{1} \cos \left(\lambda_{F} x\right)+C_{2} \sin \left(\lambda_{F} x\right)+C_{3} \cosh \left(\lambda_{F} x\right)+C_{4} \sinh \left(\lambda_{F} x\right)
$$

and the solution of eqn. (7), is:

$$
\psi(t)=A_{1} \cos (\omega t)+A_{2} \sin (\omega t)
$$

where constants $A_{1}$ and $A_{2}$ are obtained from initial conditions.

Substitution eqn. (9) into boundary conditions, eqns. (3), yields a system of homogeneous algebraic equations in unknown constants $\mathrm{C}_{\mathrm{i}}, \mathrm{i}=1,2,3,4$ with parameter $\lambda_{f}$. However, the condition of nontrivial solution for such system leads to the frequency equation as:

$$
A_{11} A_{22}-A_{12} A_{21}=0
$$

where:

$$
\begin{aligned}
& A_{11}=-\xi_{1}-\alpha_{1} \xi_{2}+\alpha_{2} \xi_{4} \\
& A_{12}=\xi_{3}-\alpha_{2} \xi_{2}+\alpha_{1} \xi_{4} \\
& A_{21}=\eta_{1}+\alpha_{1} \eta_{2}+\alpha_{2} \eta_{4} \\
& A_{22}=\eta_{3}+\alpha_{2} \eta_{2}+\alpha_{1} \eta_{4} \\
& \alpha_{1}=\frac{k_{R 0} L}{2 E I \lambda_{f}}-\frac{E I \lambda_{f}^{3}}{2 k_{T 0} L^{3}} \text { and } \alpha_{2}=\frac{k_{R 0} L}{2 E I \lambda_{f}}+\frac{E I \lambda_{f}^{3}}{2 k_{T 0} L^{3}} \\
& \xi_{1}=\cos \left(\lambda_{f}\right)+\left(K_{T L} / \lambda_{f}^{3}\right) \sin \left(\lambda_{f}\right) \\
& \xi_{2}=\sin \left(\lambda_{f}\right)-\left(K_{T L} / \lambda_{f}^{3}\right) \cos \left(\lambda_{f}\right) \\
& \xi_{3}=\cosh \left(\lambda_{f}\right)-\left(K_{T L} / \lambda_{f}^{3}\right) \sinh \left(\lambda_{f}\right) \\
& \xi_{4}=\sinh \left(\lambda_{f}\right)-\left(K_{T L} / \lambda_{f}^{3}\right) \cosh \left(\lambda_{f}\right) \\
& \eta_{1}=\left(K_{R L} / \lambda_{f}\right) \cos \left(\lambda_{f}\right)-\sin \left(\lambda_{f}\right) \\
& \eta_{2}=\left(K_{R L} / \lambda_{F}\right) \sin \left(\lambda_{F}\right)+\cos \left(\lambda_{F}\right) \\
& \eta_{3}=\left(K_{R L} / \lambda_{f}\right) \cosh \left(\lambda_{f}\right)+\sinh \left(\lambda_{f}\right) \\
& \eta_{4}=\left(K_{R L} / \lambda_{f}\right) \sinh \left(\lambda_{f}\right)+\cosh \left(\lambda_{f}\right)
\end{aligned}
$$

and the restraints stiffness parameters are:

$K_{T 0}=\frac{k_{T 0} L^{3}}{E I} \quad$ and $\quad K_{T L}=\frac{k_{T L} L^{3}}{E I}$

$K_{R 0}=\frac{k_{R 0} L}{E I} \quad$ and $\quad K_{R L}=\frac{k_{R L} L}{E I}$

The frequency equation (11) can be solved using any proper iterative technique to obtain the frequency parameters $\lambda_{\mathrm{fm}}$, where $\mathrm{m}=1,2, \ldots$ is the mode number, hence the natural frequency $\omega_{\mathrm{m}}$ can be calculated by means of eqn. (8). 
The normalized mode function is obtained assuming $\mathrm{C}_{1}$ $=1$ then, the values of the other three constants can be obtained in terms of $\alpha_{1}$ and $\alpha_{2}$. The m-mode function is obtained as:

$$
\begin{aligned}
\phi_{m}(x)= & \sin \lambda_{f m} x+\left(\alpha_{2} \alpha_{o}-\alpha_{1}\right) \cos \lambda_{f m} x-\alpha_{o} \sinh \lambda_{f m} x \\
& +\left(\alpha_{2}-\alpha_{1} \alpha_{o}\right) \cosh \lambda_{f m} x
\end{aligned}
$$

where: $\alpha_{o}=\frac{A_{11}}{A_{12}}$

Substituting eqn. (10) and eqn. (18) into eq. (5), the lateral vibration for linear case of a beam resting on linear elastic foundations is obtained as:

$$
y_{m}(x, t)=\sum\left(\begin{array}{l}
\sin \lambda_{f m} x+ \\
\left(\alpha_{2} \alpha_{o}-\alpha_{1}\right) \cos \lambda_{f m} x \\
-\alpha_{o} \sinh \lambda_{f m} x+ \\
\left(\alpha_{2}-\alpha_{1} \alpha_{o}\right) \cosh \lambda_{f m} x
\end{array}\right)\left(A \cos \omega_{m} t+B \cos \omega_{m} t\right)
$$

Constants $A$ and $B$ are obtained from initial condition and orthogonality properties of mode functions.

\subsection{Solution of the Nonlinear Vibration Equation}

The linear m-mode function is employed in the nonlinear vibration equation to obtain the solution of nonlinear case. The nonlinear vibration is assumed as:

$$
y(x, t)=y_{o} \phi_{m}\left(\lambda_{f m} x\right) \psi(t)
$$

Substitution of eqn. (20) into eqn. (2) and integrating over the $\mathrm{x}$-domain leads to:

$$
\frac{\partial^{2} \psi}{\partial t^{2}}+\gamma_{1 m} \psi(t)+\gamma_{2 m} \psi^{3}(t)=0
$$

where:

$$
\gamma_{1 m}=\omega_{m}^{2}+\frac{P_{o}}{\mu L^{2}} \frac{\int_{0}^{1} \phi^{\prime \prime} d x}{\int_{0}^{1} \phi_{m} d x}
$$

$\gamma_{2 m}=\frac{k_{2} L^{2} y_{o}^{2}}{\mu} \frac{\int_{0}^{1} \phi_{m}^{3} d x}{\int_{0}^{1} \phi_{m} d x}-\frac{E A y_{o}^{2}}{2 \mu L^{2}}\left\{\int_{0}^{1}\left(\phi_{m}^{\prime}\right)^{2} d x\right\} \frac{\int_{0}^{1} \phi_{m}^{\prime \prime} d x}{\int_{0}^{1} \phi_{m} d x}$

where $\omega_{\mathrm{m}}$ is the linear m-mode natural frequency. Integrating eqn. (21) with respect to time and assuming at $t=0, \psi=1$ and $d \psi / d t=0$, eqn. (21) may be rewritten as:

$$
\left\{\frac{d \psi}{d\left(\rho_{m} t\right)}\right\}^{2}=\left(1-\psi^{2}\right)\left(k_{m}^{2} \psi^{2}-k_{m}^{2}+1\right)
$$

where:

$$
\rho_{m}^{2}=\gamma_{1 m}+\gamma_{2 m} \text { and } k_{m}^{2}=\frac{\gamma_{2 m}}{2 \rho_{m}^{2}}
$$

Substituting $\psi(\mathrm{t})=\cos (\varphi)$, where $\varphi=\varphi(\mathrm{t})$ into equation (23), one gets: $t \rho_{m}=\int_{0}^{\varphi} \frac{d \varphi}{\sqrt{1-k_{m}^{2} \sin ^{2}(\varphi)}}$.

The integration of eqn. (25) is the elliptic integral of the first kind, its inversion yields the Jacobi elliptic function; $\operatorname{cn}\left[\rho_{m} t, k_{m}\right]$.

Then, the variation in the lateral displacement of the beam at any location with time can be expressed as:

$\psi_{m}(t)=c n\left[\rho_{m} t, k_{m}\right]$.

The period of the Jacobi elliptic function is defined by the complete elliptic integral:

$T_{m}=\frac{4}{\rho_{m}} \int_{0}^{\pi / 2} \frac{d \varphi}{\sqrt{1-k_{m}^{2} \sin ^{2}(\varphi)}}$.

Then, the natural frequency $\Omega$ for m-mode in nonlinear vibration is:

$\Omega_{m}=\frac{2 \pi}{T_{m}}$

\subsection{Verification of Present Solution}

To verify the obtained solutions, values of the frequency parameter calculated using the present solution and those obtained from numerical [1] and semi-analytical methods [10] are shown in Table $\mathbf{1}$ for conventional support condition. It is obvious that the obtained results are in close agreement with results obtained from FEM and semianalytical method. Also, it is found that value of 1E5 for restraint stiffness parameter $\mathrm{K}$ is enough to represent absolutely rigid condition (no movement)

\section{NUMERICAL RESULTS}

The derived expressions are used to study the influence of different parameters on the natural frequency of the beamfoundation system. However, the natural frequency of the system increases as the overall stiffness of the system increases. The overall stiffness of the system depends on the flexural stiffness of the beam, the stiffness of the foundation, the stiffness of the elastic end restraints. In the case of compression axial load, the lateral component of the axial load in the deformed configuration is in the opposite direction of the resultant restoring force resulted from system overall stiffness and in the same direction in case of axial tension load. Therefore, as the compressive axial force increases, the resultant of the total restoring force decreases, and the natural frequency of the system decreases. As the magnitude of the axial compression load approaches a certain critical value, its lateral component compensates the effect of the system restoring force and the system transforms to aperiodic one approaching asymptotically the equilibrium deformed configuration. Indeed, this critical value is the buckling load of the beam-soil system.

On the other hand, in case of initial axial tension, it causes an increase in the vibration frequency of the system. Moreover, as the vibration amplitude increases, the 
Table 1. Frequency Parameter $\lambda_{f m}$ for Linear Vibration

\begin{tabular}{|c|c|c|c|c|c|c|c|c|c|}
\hline \multicolumn{4}{|c|}{ Elastic End Restraints Stiffness } & \multicolumn{4}{|c|}{ Mode (m) } & \multirow{2}{*}{ Analysis } & \multirow{2}{*}{ Supports } \\
\hline $\mathbf{K}_{\mathrm{T} 0}$ & $\mathbf{K}_{\mathrm{TL}}$ & $\mathbf{K}_{\mathrm{R} 0}$ & $\mathbf{K}_{\mathrm{RL}}$ & 1 & 2 & 3 & 4 & & \\
\hline $1 \mathrm{E} 5$ & $1 \mathrm{E} 5$ & $1 \mathrm{E} 5$ & 0 & 3.924 & 7.061 & 10.191 & 13.312 & Present & \multirow{3}{*}{ Clamped - Pinned } \\
\hline $1 \mathrm{E} 5$ & $1 \mathrm{E} 5$ & $1 \mathrm{E} 5$ & 0 & 3.927 & 7.07 & 10.21 & 13.352 & {$[1]$} & \\
\hline $1 \mathrm{E} 5$ & $1 \mathrm{E} 5$ & $1 \mathrm{E} 5$ & 0 & 3.93 & 7.075 & 10.216 & 13.361 & {$[10]$} & \\
\hline $1 \mathrm{E} 5$ & $1 \mathrm{E} 5$ & $1 \mathrm{E} 5$ & $1 \mathrm{E} 5$ & 4.725 & 7.839 & 10.963 & 14.072 & Present & \multirow{3}{*}{ Clamped-Clamped } \\
\hline $1 \mathrm{E} 5$ & $1 \mathrm{E} 5$ & $1 \mathrm{E} 5$ & $1 \mathrm{E} 5$ & 4.73 & 7.782 & 11.013 & 14.155 & {$[1]$} & \\
\hline 1E5 & $1 \mathrm{E} 5$ & $1 \mathrm{E} 5$ & $1 \mathrm{E} 5$ & 4.694 & 7.794 & 10.917 & 14.04 & [10] & \\
\hline 1E5 & $1 \mathrm{E} 5$ & 0 & 0 & 3.141 & 6.282 & 9.416 & 12.546 & Present & \multirow{2}{*}{ Pinned -Pinned } \\
\hline 1E5 & $1 \mathrm{E} 5$ & 0 & 0 & 3.141 & 6.283 & 9.425 & 12.566 & {$[1]$} & \\
\hline
\end{tabular}

[1] Naidu using FEM.

[10] Wang Using Fourier transforms.

stretching due to deformed configuration produces an axial tensile force which increases the frequency parameter of the system. Bearing in mind these facts, the effects of different parameters on the vibration behavior of the beam-foundation system can be predicted qualitatively.

For the case of linear vibration of a beam resting on linear elastic foundations $\left(\mathrm{P}_{\mathrm{o}}=0, \mathrm{k}_{2}=0\right.$ and stretching due to transverse vibration is neglected) values for frequency parameter $\lambda_{\mathrm{fm}}$ against different values of end restraints are shown in Table 2. It is clear that the frequency parameter increases as the stiffnesses of the end restraints increase. The frequency parameter for subsequent mode is shifted by $\pi$ for conventional end supports and by value less than $\pi$ for elastic end restrains. However, the effect of stiffness of elastic end restrains is more noticeable for frequency parameter of lower modes.
The m-frequency parameter $\lambda_{f m}$ for the nonlinear case is defined as:

$\lambda_{f m}^{4}=\frac{\mu L^{4} \Omega_{m}^{2}}{E I}$

Using dimensionless parameters with respect to geometric properties of the beam eliminates the effect of the geometric properties of the beam on the frequency parameter.

The effects of dimensionless vibration amplitude on the fundamental frequency parameter $\lambda_{f}(\mathrm{~m}=1)$ for different values of load parameter $\bar{P}_{o}$, end conditions and foundation stiffnesses $\left(\vec{k}_{l}, \bar{k}_{2}\right)$ are shown in Fig. (2). Fig. (2A) represents the case of a beam without foundation and Fig. (2B) represents the beam-foundation system. The dimensionless load parameter and foundation parameters are defined as:

Table 2. Frequency Parameter $\lambda_{f m}$ for Linear Vibration

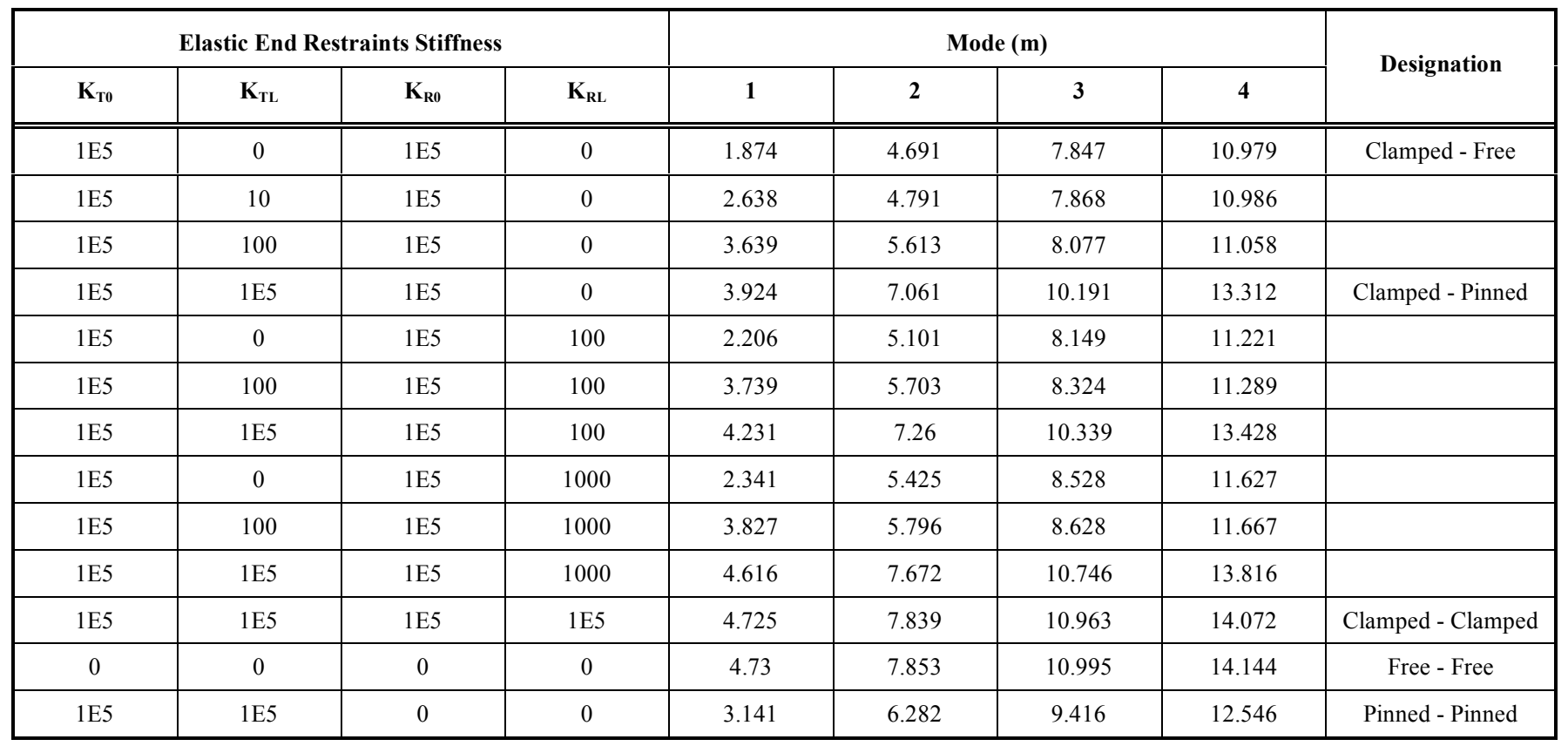


(A) Beam without foundation $\left(\bar{k}_{1}=\bar{k}_{2}=0\right)$

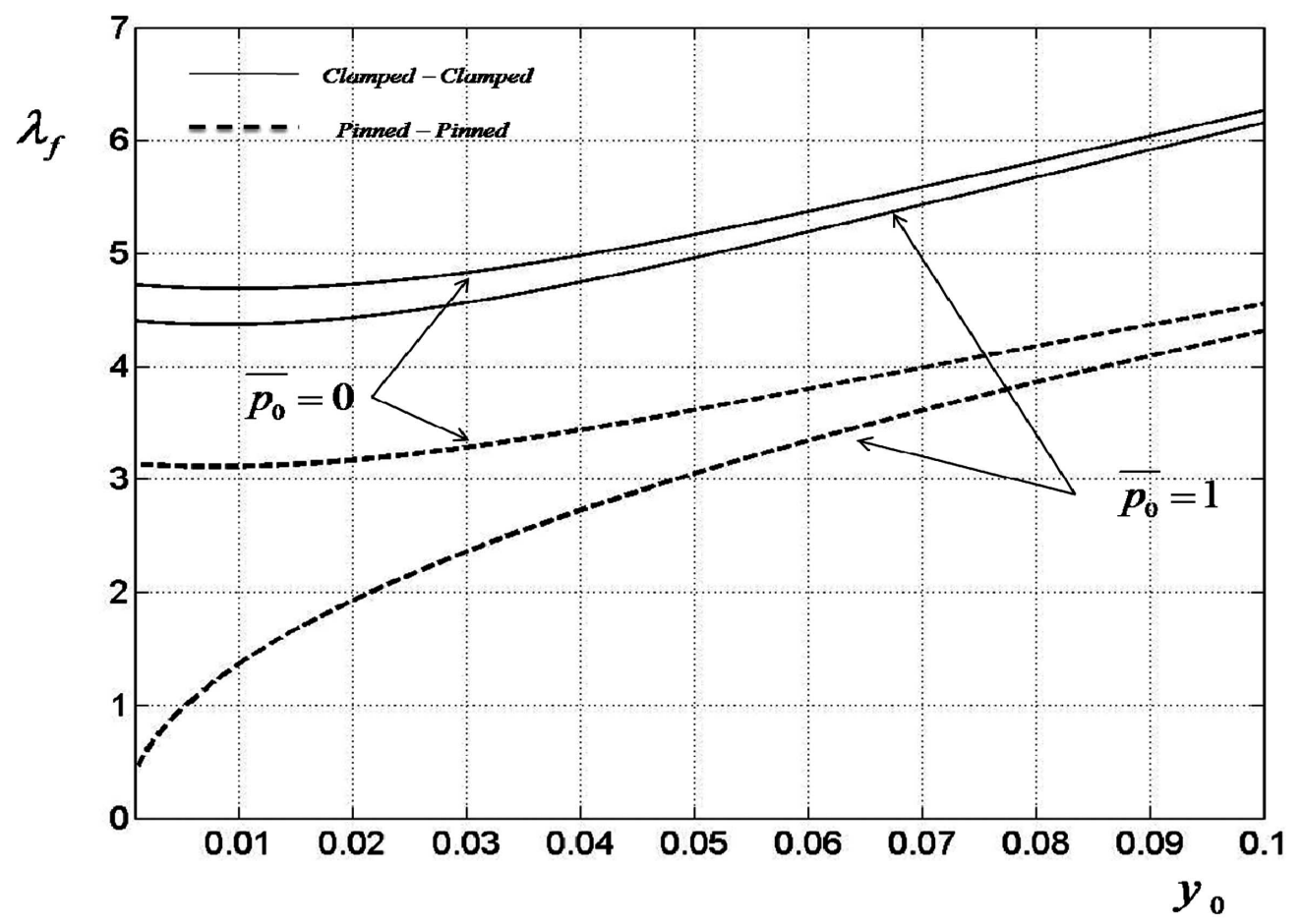

(B) Beam - foundation system $\left(\bar{k}_{1}=1 E 2\right.$ and $\left.\bar{k}_{2}=1 E 5\right)$

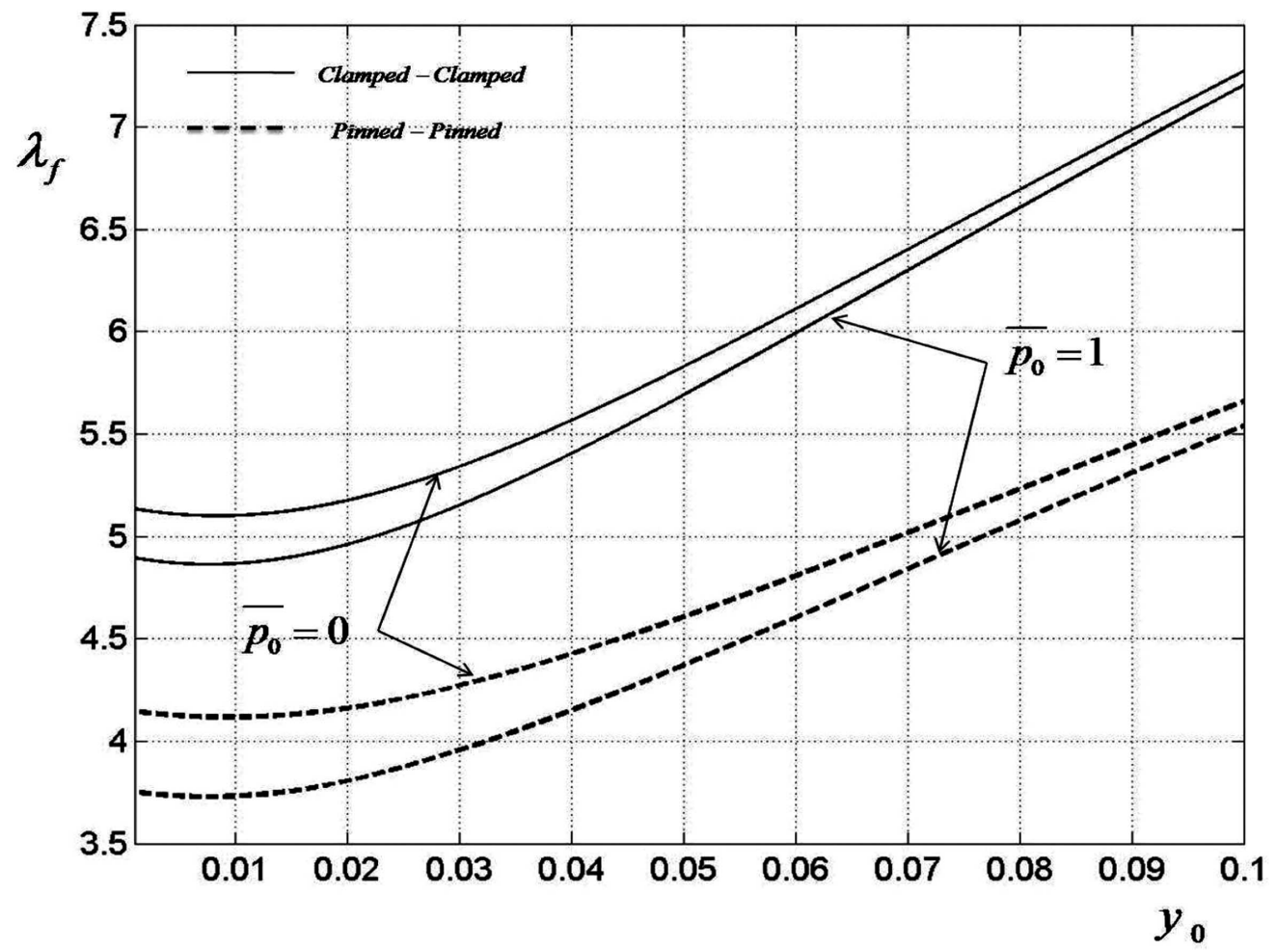

Fig. (2). Influence of dimensionless vibration amplitude $y_{o}$ on frequency Parameter $\lambda_{f}$.

$\bar{P}_{o}=\frac{P_{o} L^{2}}{\pi^{2} E I}$

$\bar{k}_{1}=\frac{k_{1} L^{4}}{E I}$ and $\bar{k}_{2}=\frac{k_{2} L^{6}}{E I}$
In case of beam without foundation, as the value of $\bar{P}_{o}$ approaches the buckling value, the frequency parameter approaches zero (Aperiodic system). Also, it should be noted that, the value for critical axial load (buckling load) in case of beam-foundation system is greater than its value in case of beam without foundation. 
(A) Effects of foundation parameters $\left(\bar{P}_{o}=1\right)$

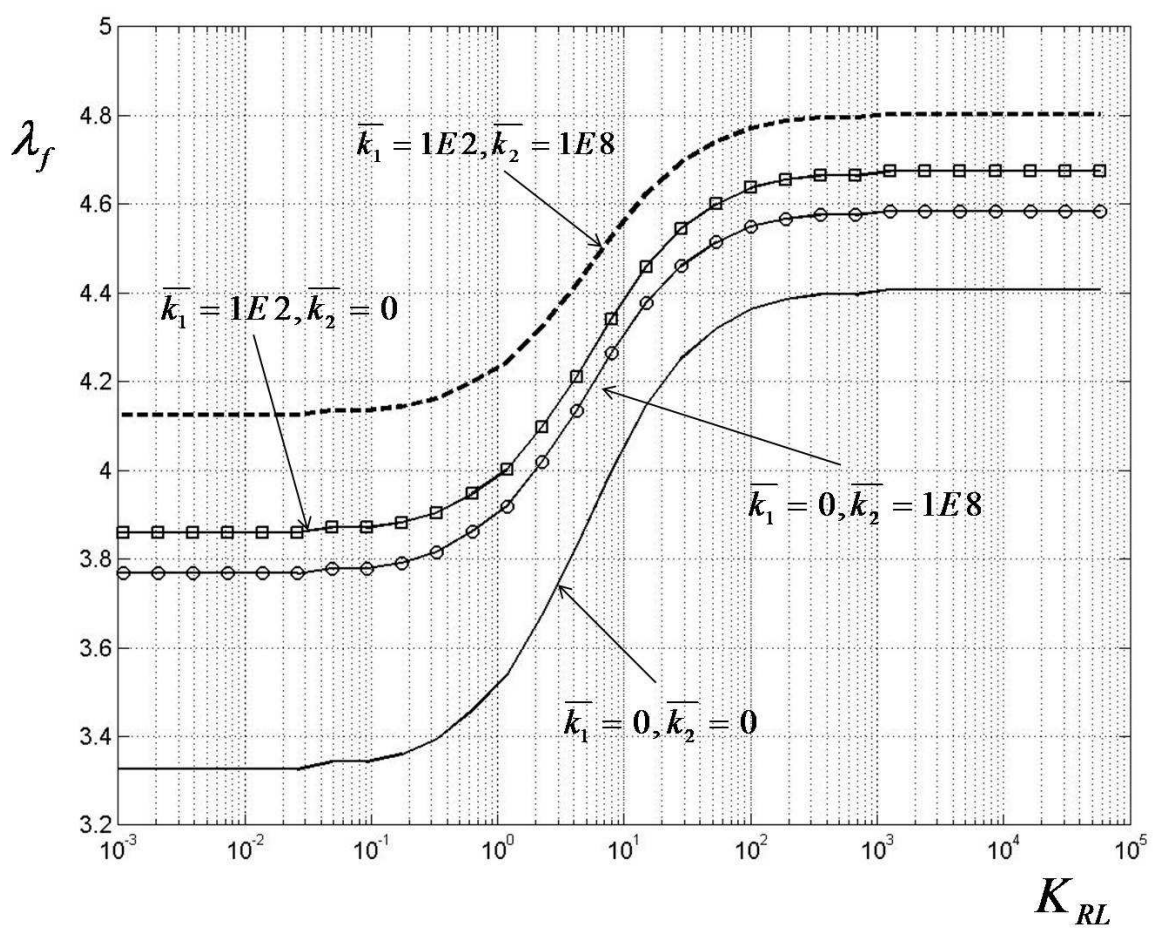

(B) Effects of load parameter $\left(\bar{k}_{1}=1 E 2\right.$ and $\left.\bar{k}_{2}=1 E 5\right)$

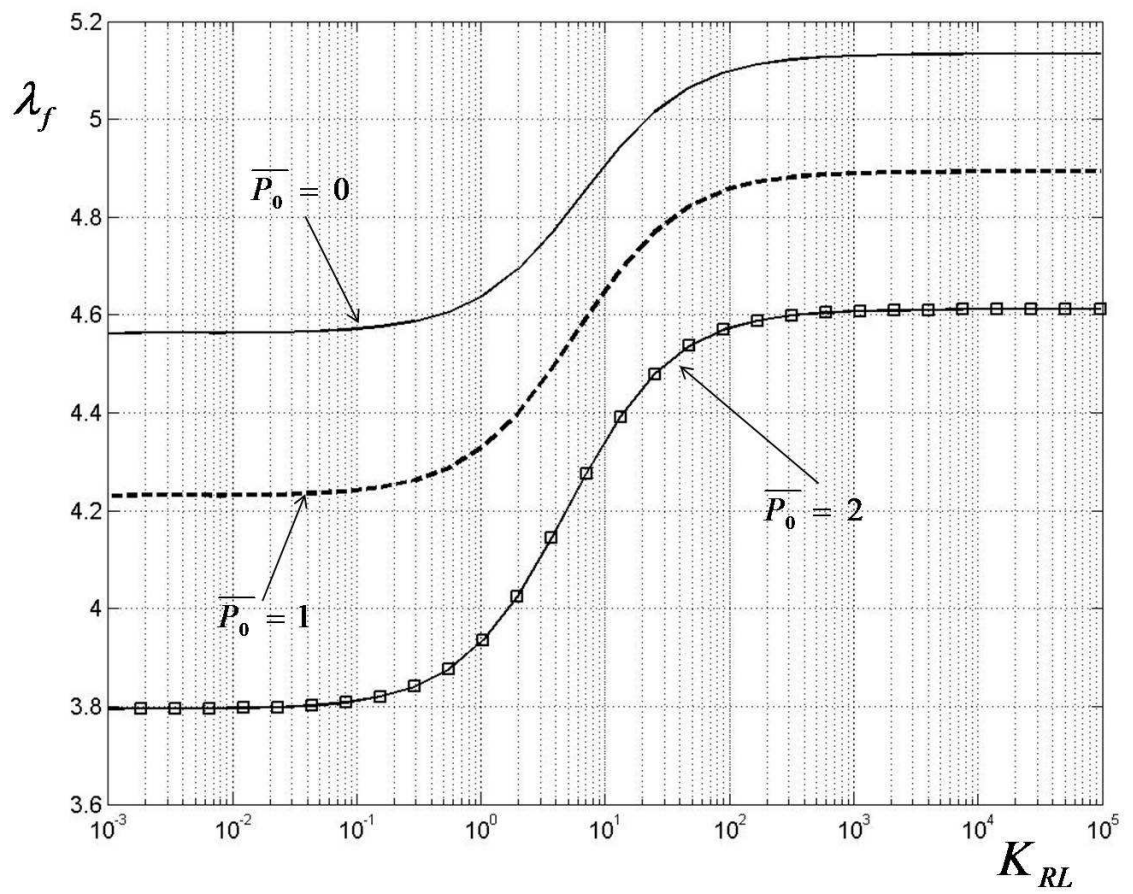

Fig. (3). Influence of rotational stiffness parameter on frequency parameter $\lambda_{f}\left(K_{T 0}=K_{T L}=K_{R 0}=1 E 5\right)$.

Fig. (3) shows the influences of elastic rotational stiffness at one end on frequency parameter $\lambda_{f}$ for different values of foundation parameters in Fig. (3A) and for different values of load parameter in Fig. (3B). It is obvious that the frequency parameter increases as the system stiffness increases and as the load parameter decreases. The limiting values of the frequency parameter represent the case of conventional end conditions. 
(A) Effects of rotation stiffness $\left(K_{T L}=1 E 5\right)$

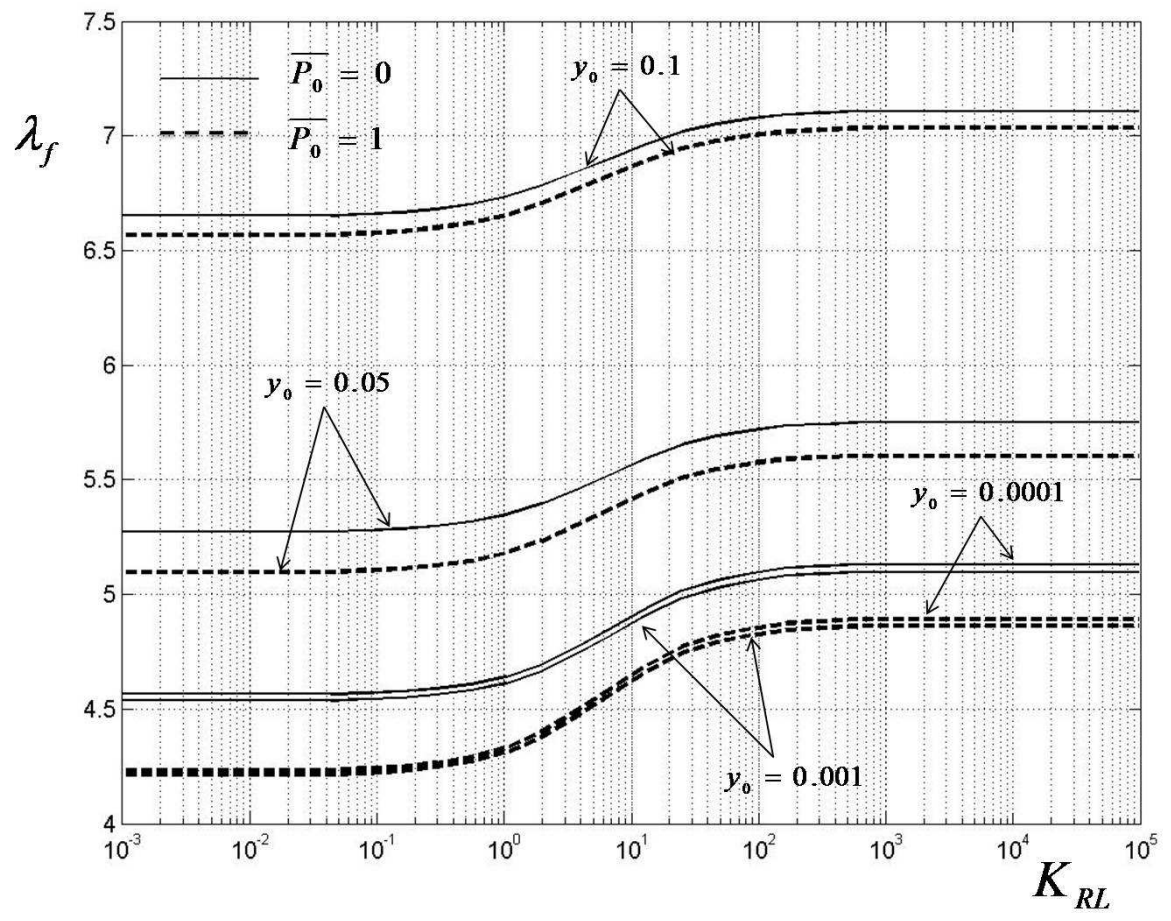

(B) Effects of lateral translation stiffness $\left(K_{R L}=1 E 5\right)$

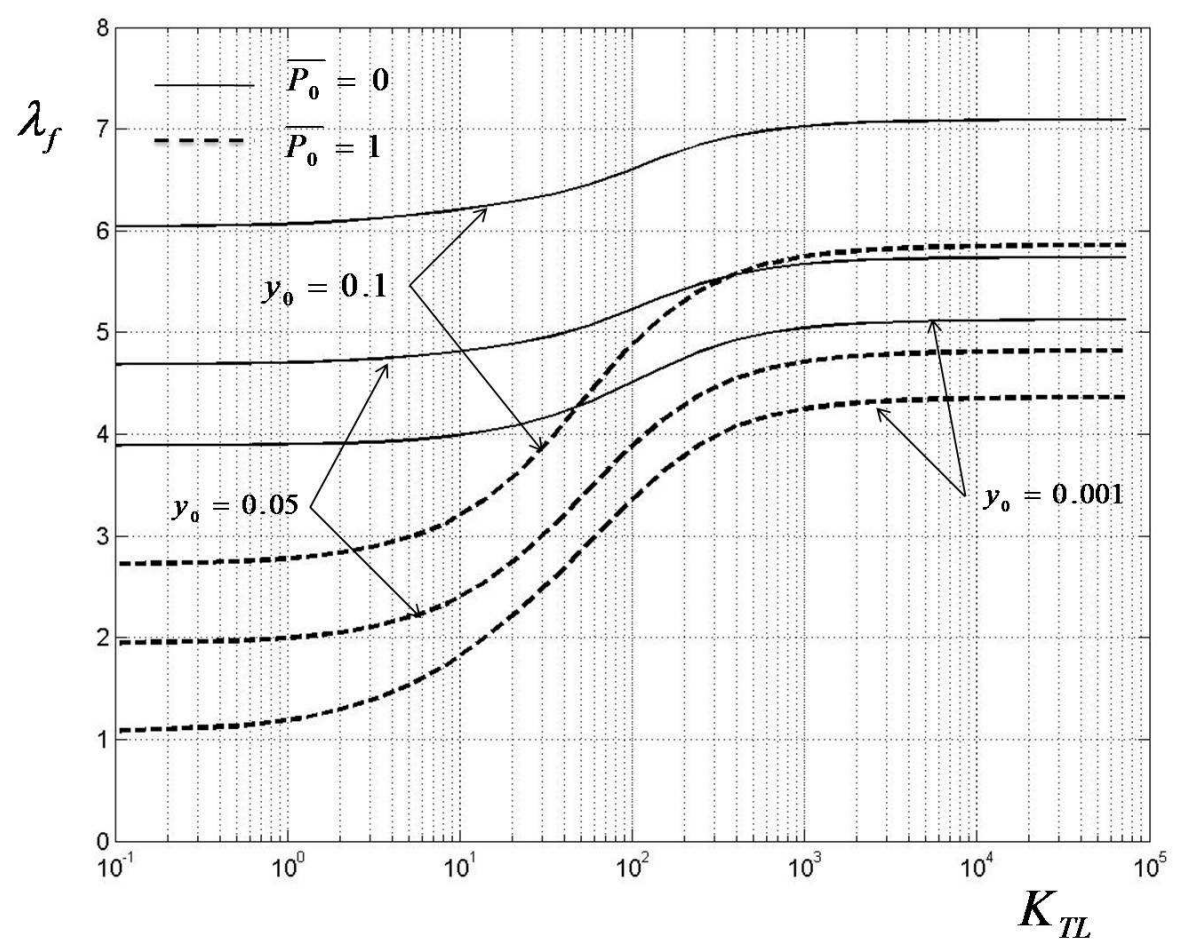

Fig. (4). Influences of vibration amplitude and load parameter on frequency parameter $\lambda_{f}\left(K_{T 0}=K_{R 0}=1 E 5\right.$ and $\left.\bar{k}_{1}=1 E 2, \bar{k}_{2}=1 E 5\right)$.

Fig. (4) depicts the influences of dimensionless vibration amplitude $y_{o}$ and elastic stiffness at one end on the frequency parameter. Fig. (4A) represents the effects of elastic rotation stiffness at one end and Fig. (4B) represents the effects of elastic lateral translation stiffness. 
(A) Beam clamped at one end $\left(K_{R 0}=1 E 5\right)$

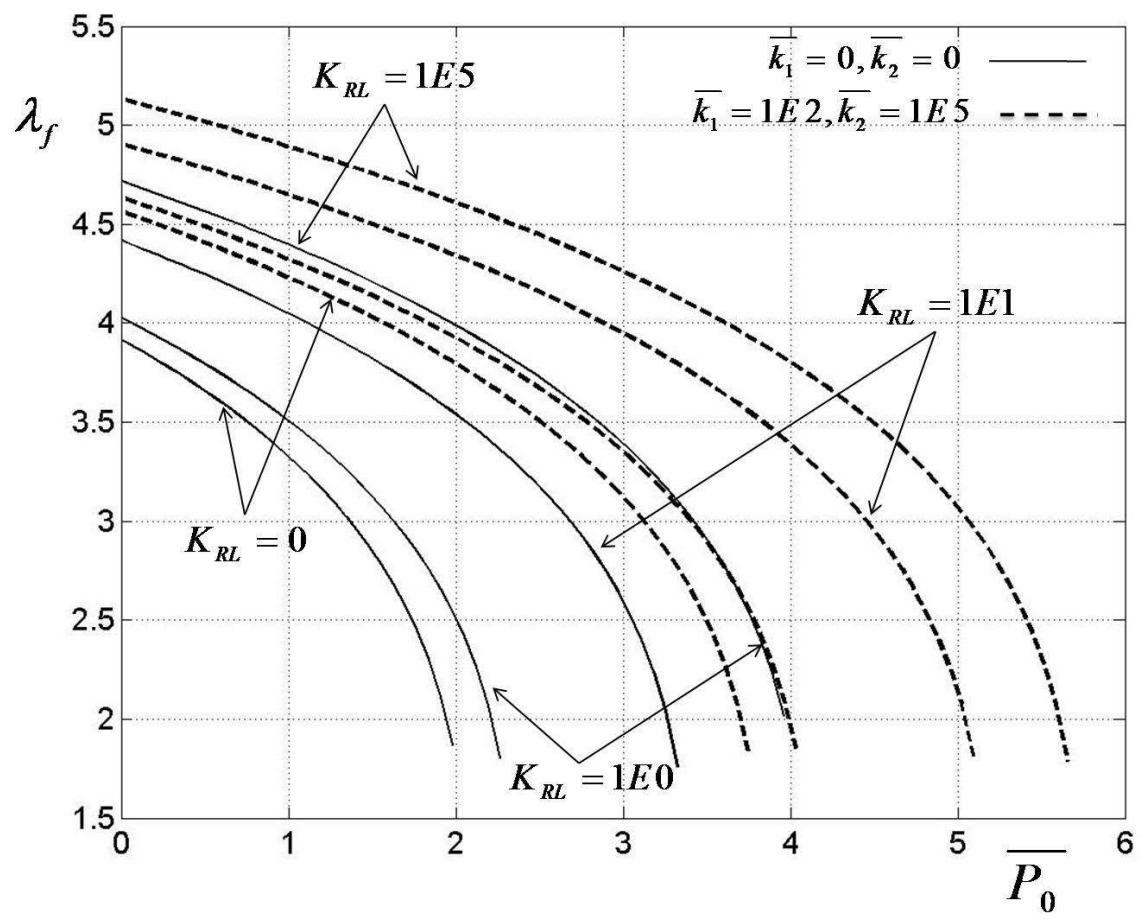

(B) Beam pinned at one end $\left(K_{R 0}=0\right)$

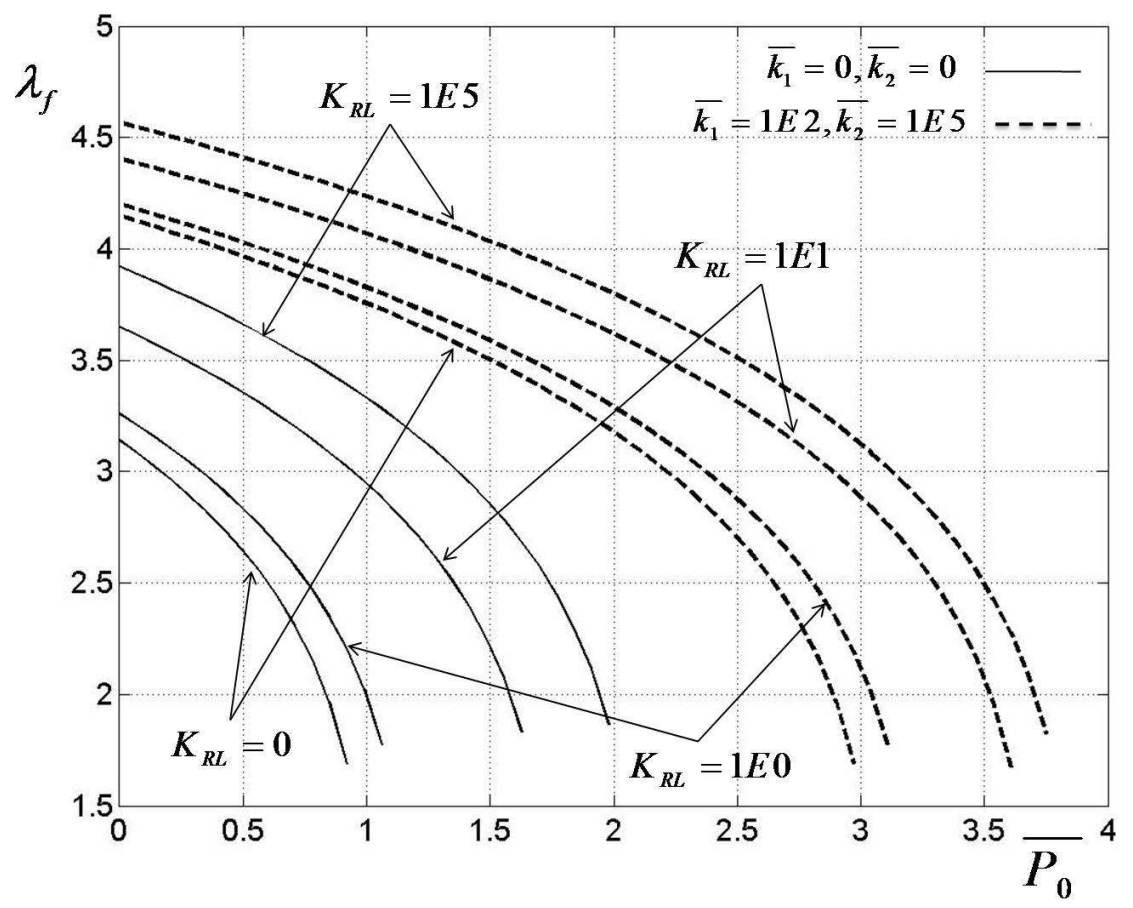

Fig. (5). Influences of load parameter and rotational stiffness on frequency parameter $\lambda_{f}\left(K_{T 0}=K_{T L}=1 E 5\right)$.

In Fig. (5), the effects of the load parameter on the frequency parameter for different end restrains are shown. The figure predicts both the frequency parameter for beamfoundation system of different characteristics and the critical (Buckling) load for such cases.
Fig. (6) shows the influence of end lateral translation stiffness parameter on the frequency parameter $\lambda_{f}$ for different values of foundation parameters, while the load parameter takes the value of Euler critical load for simply supported beam without foundation. The effect of variation 


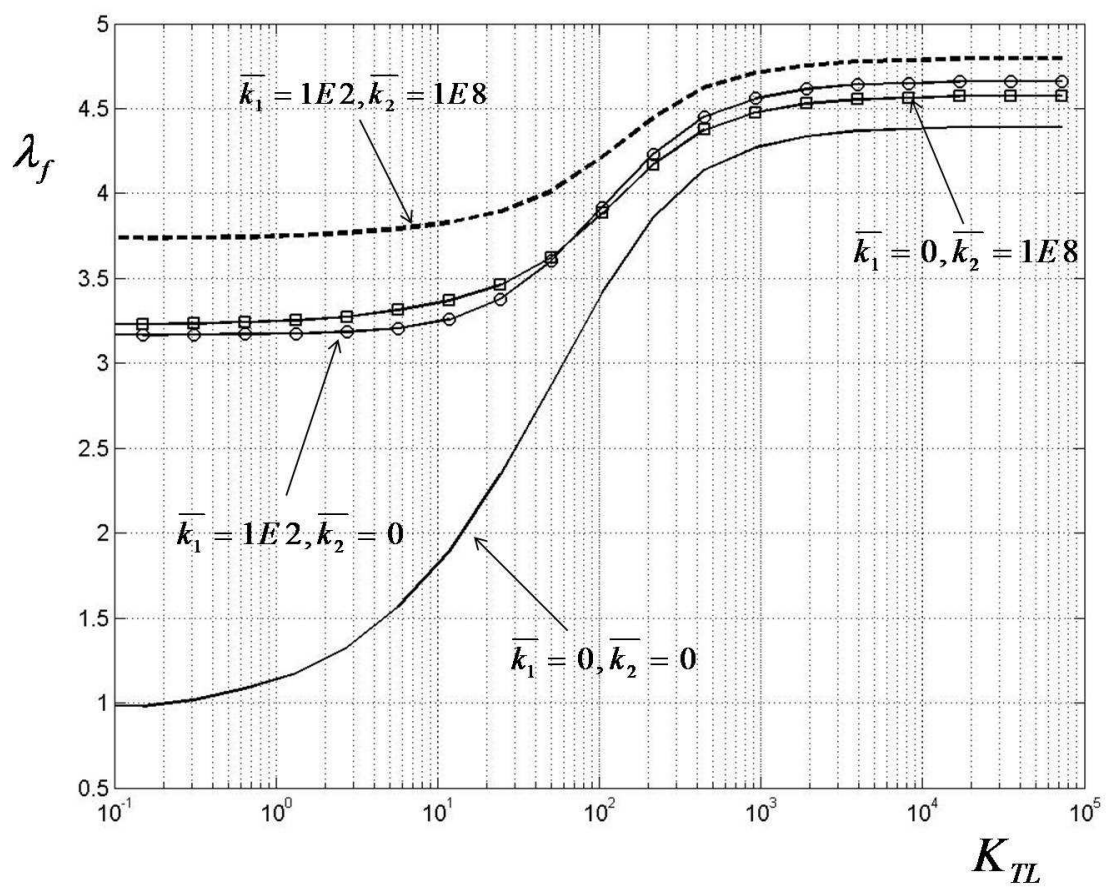

Fig. (6). Influence of lateral translation stiffness on frequency parameter $\lambda_{f}\left(\bar{P}_{o}=1\right.$ and $\left.K_{T 0}=K_{R 0}=K_{R L}=1 E 5\right)$.

of elastic end restrains stiffness is less noticeable for beams resting on foundations.

\section{CONCLUSIONS}

Analytic expressions for the natural frequencies of nonlinear vibration of an initially stressed beam with elastic end restraints resting on a nonlinear foundation are obtained. It is found that the natural frequency of the beam-foundation system increases as the overall stiffness of system increases. The overall stiffness of the system is composed of the flexural stiffness of the beam, the stiffness of elastic restraints at ends and the foundation stiffness. The natural frequency of the system decreases as the axial compression load increases. However, as the axial compression load approaches certain value, the system transforms to aperiodic system and approaches asymptotically the deformed equilibrium configuration. Indeed, this critical value is called the buckling load. The stretching due to lateral vibration amplitude releases the effect of axial compression load, leading to an increase in the natural frequency of the system. Furthermore, the shifting of higher modes in case of elastic end restraints is smaller than $\pi$ while it is approximately equal $\pi$ for conventional end conditions.

\section{ACKNOWLEDGEMENT}

Declared none.

\section{CONFLICT OF INTEREST}

The authors confirm that this article content has no conflicts of interest.

\section{REFERENCES}

[1] Naidu NR, Rao GV. Free vibration and stability behavior of uniform beams and columns on nonlinear elastic foundation. Comput Struct 1996; 58(6): 1213-5.

[2] Thambiratnam D, Zhuge Y. Free vibration analysis of beams on elastic foundations. Comput Struct 1996; 60(6): 971-80.

[3] Ayvaz Y, Ozgan K. Application of modified Vlasov model to free vibration analysis of beams resting on elastic foundations. Sound Vib 2002; 255(1): 111-27.

[4] Chen CN. Vibration of prismatic beam on an elastic foundation by the differential quadrature element method. Comput Struct 2000; 77: 1-9.

[5] Chen CN. DQEM Vibration analysis of non-prismatic shear deformable beams resting on elastic foundations. Sound Vib 2002; 255(5): 989-99.

[6] Chen WQ, Lu CF, Bain ZG. A mixed method for bending and free vibration of beams resting on Pasternak elastic foundations. Appl Math Model 2004; 28: 877-90.

[7] Nayfeh AH, Nayfeh SA. On nonlinear modes of continuous systems. J Vib Acoust 1994; 116: 129-36.

[8] Maccari A. The asymptotic perturbation method for nonlinear continuous systems. Nonlinear Dyn 1999; 19: 1-18.

[9] Ruta P. The vibration of a non-prismatic beam on an inertial elastic half-plane. Sound Vib 2004; 275: 533-56.

[10] Wang JTS, Lin CC. Dynamic analysis of generally supported beams using Fourier series. Sound Vib 1996; 196(3): 285-93.

[11] Taha MH. Transient response of finite elastic beam on viscoelastic foundation under stochastic dynamic loads. Int J Differ Equ Appl 2002; 6/3:283-97.

[12] Taha MH, Abohadima S. Mathematical model for vibrations of nonuniform flexural beams. J Eng Mech 2008; 15(1): 3-11.

[13] Abohadima S, Taha MH. Dynamic analysis of nonuniform beams on elastic foundations. Open Appl Math J 2009; 3: 40-4. 